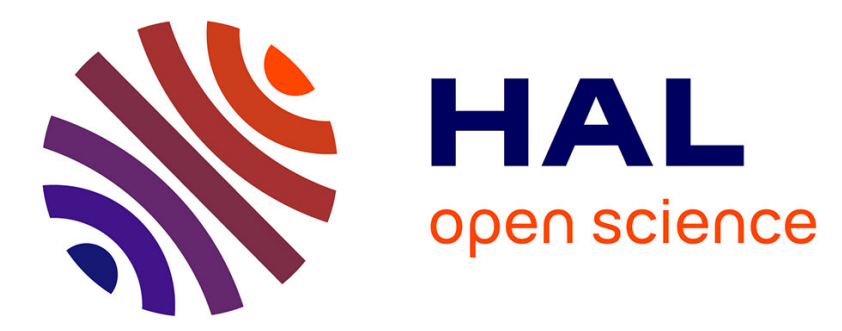

\title{
Interdisciplinarity in practice: Challenges and benefits for privacy research
}

Daniel Le Métayer, Mathias Bossuet, Fanny Coudert, Claire Gayrel, Francisco Jaime, Christophe Jouvray, Antonio Kung, Zhendong Ma, Antonio Maña

\section{- To cite this version:}

Daniel Le Métayer, Mathias Bossuet, Fanny Coudert, Claire Gayrel, Francisco Jaime, et al.. Interdisciplinarity in practice: Challenges and benefits for privacy research. Computer Law and Security Review, 2017, 33 (6), pp.864-869. 10.1016/j.clsr.2017.05.020 . hal-01660055

\section{HAL Id: hal-01660055 https://hal.inria.fr/hal-01660055}

Submitted on 9 Dec 2017

HAL is a multi-disciplinary open access archive for the deposit and dissemination of scientific research documents, whether they are published or not. The documents may come from teaching and research institutions in France or abroad, or from public or private research centers.
L'archive ouverte pluridisciplinaire HAL, est destinée au dépôt et à la diffusion de documents scientifiques de niveau recherche, publiés ou non, émanant des établissements d'enseignement et de recherche français ou étrangers, des laboratoires publics ou privés. 


\title{
Interdisciplinarity in practice: Challenges and benefits for privacy research
}

Daniel Le Métayer (Inria, Université de Lyon), Mathias Bossuet (Thales), Fanny Coudert (KU Leuven), Claire Gayrel (University of Namur), Francisco Jaime (UMA), Christophe Jouvray (Trialog), Antonio Kung (Trialog), Zhendong Ma (AIT), Antonio Maña (UMA)

Computer Law \& Security Review

Volume 33, Issue 6, December 2017, Pages 864-869

\begin{abstract}
The goal of this paper is to draw the lessons learned from a project that involved security systems engineers, computer scientists, lawyers and social scientists. Since one of the goals of the project was to propose actual solutions following the privacy by design approach, its aim was to go beyond multidisciplinarity and build on the variety of expertise available in the consortium to follow a true interdisciplinary approach. We present the challenges before describing the solutions adopted by the project to meet them and the outcomes and benefits of the approach. We conclude with some lessons to be drawn from this experience and recommendations for future interdisciplinary projects.
\end{abstract}

\section{Multidisciplinarity and interdisciplinarity}

Multidisciplinarity and interdisciplinarity are often praised in official statements and put forward in calls for projects but one must admit that the distance is great between rhetoric and reality. As noted in a report dedicated to the European FET programme ${ }^{1}$, "there is a discrepancy between willingness to develop multidisciplinary research at strategic level and the reality in the field". In many areas however, interdisciplinarity is not really an option, it is a prerequisite to be able to deal with the complexity and the multiple dimensions of the problems to be solved. Privacy is one of these areas because privacy protection is a legal right that can be jeopardized, put into question, or enhanced by many technical, social and economic evolutions. An interdisciplinary approach is necessary not only to design effective privacy protection instruments but also to understand the complexities of the concept itself, its multiple facets and interpretations. However, setting up a successful collaboration between disciplines which are as varied and remote as computer science, law and social sciences is a challenge in itself.

\footnotetext{
${ }^{1}$ Multidisciplinary research in FET, V. Gayraud, FET Trainee Report, 24/11/05.
} 
First, a distinction should be made between multidisciplinarity and interdisciplinarity even if these words are sometimes used interchangeably. Different definitions have been proposed for these terms ${ }^{2}$ in the literature ${ }^{3}$. In this paper, we use them in the following sense:

- Multidisciplinarity is the basic level of collaboration between disciplines. It refers to different disciplines "working on a problem in parallel or sequentially, and without challenging their disciplinary boundaries." ${ }^{4}$ Each team produces its own results, without any specific integration effort with other disciplines even if the results are exchanged and discussed in order to enhance the global understanding of the object under study.

- Interdisciplinarity "brings about the reciprocal interaction between disciplines", "in order to generate new common methodologies, perspectives, knowledge." The interdisciplinary approach is more ambitious as it aims to develop research results integrating contributions from different disciplines, which does not exclude specific benefits in each discipline (new ways of considering a question, methodological transfers, etc.).

In fact, multidisciplinarity and interdisciplinarity should not be seen as two entirely distinct categories but rather as the two extremities of a continuum and a project involving several disciplines can generally be placed somewhere in between.

The goal of this paper is to draw the lessons learned from the PARIS European project that involved security systems engineers, computer scientists, lawyers and social scientists. Since one of the goals of the project was to propose actual solutions following the privacy by design approach, its aim was to go beyond multidisciplinarity and build on the variety of expertise available in the consortium to follow a true interdisciplinary approach. We first briefly describe the context and the objectives of the project in Section 2 before presenting the challenges to be addressed to follow the interdisciplinary approach in Section 3. In Sections 4 and 5 we describe respectively the solutions adopted by the partners to meet these challenges and the outcomes and benefits of the approach. We conclude in Section 6 with some lessons to be drawn from this experience and recommendations for future interdisciplinary projects.

\section{Context: interdisciplinarity as a conditions of success}

The context of the project, called PARIS ${ }^{5}$, was the increasing adoption of surveillance infrastructures all over the world and the need to protect the right of citizens for privacy, justice and freedom. Two key notions were put forward in the project, consistently with the new European General Data Protection Regulation: privacy by design and accountability (including Privacy Impact Assessment). More precisely, the main goal of the project was to propose a theoretical generic framework for the design of privacy preserving and accountable

\footnotetext{
${ }^{2}$ And others such as pluridisciplinarity, crossdisciplinarity, or transdisciplinarity.

${ }^{3}$ For example in: Ten cheers for interdisciplinarity: the case for interdisciplinary knowledge and research, $\mathrm{M}$. Nissani, The Social Science Journal, Vol. 34, No 2, pp. 201-216, 1997. Multidisciplinarity, interdisciplinarity and transdisciplinarity in health research, services, education and policy: 1. Definitions, objectives, and evidence of effectiveness, B.C.K. Choi, A.W.P. Pak, Clin. Invest. Med., Vol. 29, No 6, pp.351-364, 2006. Multidisciplinary research in FET, V. Gayraud, FET Trainee Report, 24/11/05.

${ }^{4}$ Multidisciplinarity, interdisciplinarity and transdisciplinarity in health research, services, education and policy : 1. Definitions, objectives, and evidence of effectiveness, B.C.K. Choi, A.W.P. Pak, Clin. Invest. Med., Vol. 29, No 6, pp.351-364, 2006.

${ }^{5}$ PARIS is an acronym for "PrivAcy pReserving Infrastructure for Surveillance".
} 
surveillance systems together with guidelines to define specialized frameworks suitable in different contexts (e.g. for different countries or types of technologies). Two use cases were considered, the first one being based on biometrics and the second one on video analysis and forensics search technologies.

The main challenge of the project was to provide tools to try to reconcile the operational objectives of a surveillance system with the necessity to preserve the fundamental rights of the people subject to such surveillance. Considering the variety of considerations and requirements to be taken into account (technical, legal, social, etc.), interdisciplinarity was not a choice for this project: it was a condition for its success. The composition of the consortium reflected this need, including computer scientists (from industry ${ }^{6}$ and research ${ }^{7}$ ), lawyers ${ }^{8}$ and social scientists ${ }^{9}$. But interdisciplinarity is a challenge in itself especially when it concerns disciplines with very different histories, cultures and practices (research development, assessment, collaborations, etc.). These challenges are discussed in the next section.

\section{Challenges raised by interdisciplinarity}

Even when they have a common object of study, different disciplines usually take very different perspectives and follow different approaches, with different objectives and different ways to assess their results. All these differences can become sources of misunderstanding, frustration, and dispersion and may ultimately cause the failure of an interdisciplinary project. Privacy in itself is a challenging topic to this respect because it is a multifaceted and evolving notion, understood in different ways by different disciplines and even within each disciplinary field.

Privacy is a legal notion which has been interpreted and protected in various ways depending on times and cultures (from Warren and Brandeis "right to be let alone", to Westin "claim of individuals, groups or institutions to determine for themselves when, how, and to what extent information about them is communicated to others" and the current focus on "user empowerment"). Sociologists often refer to privacy as an implicit norm that regulates and facilitates social interactions. Privacy is thus linked to notions of unpredictability, originality and liberty in general. In that perspective, privacy becomes a political stake very related to the control versus freedom, or integration versus diversity tensions and can be regarded as a political condition for the vitality of our democracies. In computer science, the word "privacy" is often taken in a restricted sense as the compliance with a set of properties such as confidentiality, anonymity, or denialability. As an illustration, the "Common Criteria for Security", the most internationally recognized standard for security evaluation, include a specific chapter on privacy and defines it in terms of four properties: anonymity, pseudonymity (possibility to use pseudonyms instead of identity attributes), non-traceability (impossibility to link several actions performed by a single user) and non-observability (impossibility to know that an actor has performed a given action).

\footnotetext{
${ }^{6}$ Trialog (France), Thales Communication \& Security (France) and Visual Tools (Spain).

${ }^{7}$ AIT Austrian Institute of Technology (Austria), Inria (France), and Universidad de Malaga (Spain).

${ }^{8}$ Katholieke Universiteit Leuven (Belgium), Facultés Universitaires Notre-Dame de la Paix de Namur (Belgium).

${ }^{9}$ Facultés Universitaires Notre-Dame de la Paix de Namur (Belgium).
} 
Roughly speaking, one could say that both computer science and sociology take a rather practical or objective approach: computer science explores all the possibilities from the technological point of view and sociology takes the point of view of society (impact of technologies, acceptance, rejection, etc.). On the other hand, lawyers tend to place the debate at the level of principles and rules. Needless to say, any ambitious project on privacy needs to encompass all these dimensions.

The first difficulty has to do with terminology: for example the legal terminology, even if it seems to refer to a technical vocabulary (e.g. privacy by design, privacy risk analysis, anonymization, pseudonymization, etc.), is often misunderstood by technical experts because these terms are generally used in a loose, and sometimes misleading way. Sociologists face similar difficulties, with terms such as "actor", "agent", "user", or framework" for example. In fact, the difficulties in mutual understanding are even greater with terms that are used in different ways by different disciplines (or with subtle variations that the partners may take time to realize) than with terms specific to a discipline.

Another major source of difficulty is the different forms of normativities introduced respectively by law, technology and society. These sources of normativities are often conflicting, but they can also be converging if appropriate interdisciplinary cooperation takes place. For example new approaches in computer science such as "privacy by design" and "accountability" seem quite promising because they introduce the idea of a certain liability by data controllers and therefore establish a practical link between technology and law.

Other, deeper sources of divergences may come from the differences in terms of methodologies and research objectives. For example, a potential cause of conflict between computer scientists on one hand and lawyers and social scientists on the other hand, is the mechanization objective. Indeed, it is a natural trend for a computer scientist to exploit as much as possible the possibilities of the computers and to devise systems that are as generic and automatic as possible. On the other hand, lawyers and social scientists emphasize the need to work on a case-by-case basis, in order to take into account all the complexities and specificities of real life situations. Automation, trying to encompass a wide variety of cases, often leads to oversimplification, which cannot be acceptable from legal or sociological points of view.

These differences in terms of terminology and objectives are also reflected in the research methodology itself, especially with respect to the importance granted to discussion, negotiation, and argumentation. Arguing, debating, developing ideas and conceptualizing are common for lawyers and social scientists whereas computer scientists are generally more inclined to use direct and terse communication styles. These differences of communicating style appear both in oral and written communications ${ }^{10}$. As worded by Bruno Latour ${ }^{11}$, "Scientists talk loosely about precise objects while lawyers talk precisely about loose objects". These differences of attitudes have to be understood and addressed as soon as possible to avoid painful and non-productive discussions.

\footnotetext{
${ }^{10}$ To this respect, the differences in terms of size and level of precision and detail between documents produced by computer scientists and lawyers is striking. Lawyers may find it hard to follow computer science reports written in a concise and terse way whereas computer scientists can be lost in the details and size of documents produced by lawyers.

11 « Les savants parlent mal d'objets justes, les juristes parlent juste d'objets flous. »
} 


\section{Solutions adopted by the project}

The PARIS project has been set up from the beginning with interdisciplinarity in mind and its structure reflected this ambition: rather than dedicating tasks to specific disciplinary approaches (e.g. legal view, social sciences view, computer science view), the choice was made to focus on objectives with all partners involved (even if, for each task, one of them had to take the lead for organizational requirements). This choice proved useful to foster collaboration between partners.

From the practical point of view, a large amount of time has been devoted to open discussions, especially during the first two years of the project, to reach a common understanding of the main concepts, objectives and the points of view of each discipline. These discussions took place within plenary project meetings. They were conducted in a nondirective way and without strong timing constraints in order to ensure that all points of view are expressed and all topics debated. They were followed by the preparation of reports describing the respective positions of the partners and documenting any agreement, divergence or progress towards better mutual understanding.

Between two plenary meetings, the exchanges continued through a dedicated wiki page and mutual visits between the partners. The partners also elaborated a set of shared taxonomies based on their respective areas of expertise to share their knowledge and improve their mutual understanding.

The main topics have been discussed within several iterations, allowing the participants to think about all the issues and the information exchanged during the previous step and to make new suggestions. It turned out to be very useful to use concrete examples to illustrate the different points of view. When divergences were too strong, it also happened that an internal mediator was appointed to organize point-to-point discussions to solve the issue.

\section{Main outcomes and benefits of the interdisciplinary approach}

The goal of this paper is not to describe the results of the PARIS project but we outline in this section two outcomes of the project that illustrate the benefits of the interdisciplinary approach. None of these results would have been reachable through a single-discipline or even a multidisciplinary approach.

\subsection{The SALT Framework}

One of the main deliverables of the project is a framework called SALT ${ }^{12}$ whose goal is to help owners, designers and developers of surveillance systems to take into account all relevant legal, ethical, social and technical factors. The core of this framework is a knowledge-based repository resulting from interactions with experts from different disciplines. SALT follows a questionnaire-based approach for system owners and designers

\footnotetext{
${ }^{12}$ SALT stands for Socio-ethicAl, Legal, and Technical.
} 
backed up by the knowledge repository. The three stages of the process are respectively: (1) the intention stage, (2) the design stage, and (3) the implementation stage.

The goal of the first stage is to question the opportunity of installing the surveillance system and to check the proportionality requirement. Due to the legal aspects of the questions raised at this stage, these questions should be answered by a lawyer. However, the lawyer using the SALT framework does not need to be an expert regarding data protection because he or she is guided by an interactive questionnaire submitting the appropriate questions.

The second stage ensures that all requirements are taken into account during the design process. The performance of the system is based on design restrictions and recommended design artifacts extracted from the results of the first stage. In addition, the process allows for a system design validation (using OCL validation rules extracted from the SALT elements selected during the process). Whenever one of these rules is not fulfilled, a warning message is displayed to the user. The result of this stage is a system design (typically in the form of a UML model) together with a documentation. This documentation provides information related to the design choices and their motivation.

The third stage includes a final assessment of the overall system with respect to its initial aims and legal requirements. This task is based on the implemented system, together with the system documentation generated during the design phase. Its result is an evaluation report.

The SALT framework is flexible in the sense that it can evolve over time and benefit from the input of SALT experts ${ }^{13}$. Also, the framework contributes to the accountability requirement because it can trace the assumptions used during the decision-making process and the positions of the various stakeholders.

One of the key challenges for the design of the SALT framework was to integrate the questions-based approach to address privacy and ethical issues in such a way that it is likely to generate self-questioning for the users (owner, designer, etc.) and eventually debates among stakeholders. It should also be noticed that not only engineers, but also lawyers are involved in the whole SALT process from the beginning to the end.

\subsection{Analysis of the case law of a Data Protection Authority}

A specific research has been carried out in the PARIS project in order to provide an example questionnaire for biometric systems. This research has included an extensive study of the French case law in relation to biometric systems, contributions from the Council of Europe, the Working Party 29 and literature ${ }^{14}$. There was no single, uniform and harmonized interpretation at the European level as to which surveillance technologies could be deemed acceptable or not, and the conditions under which they can be deployed. While only general guidance is available in Belgium, extensive deliberations have been issued by the CNIL ${ }^{15}$ in France regarding biometrics applications. Since 2005, the CNIL is empowered to authorize biometric systems (except those deployed following the adoption of a decree), and all

\footnotetext{
13 Each user can potentially become an expert.

${ }^{14}$ Claire Gayrel, "The principle of proportionality applied to biometrics in France, ten years of CNIL's deliberations" to appear in Computer Law and Security Review.

15 CNIL (Commission Nationale de l'Informatique et des Libertés) is the French Data Protection Authority.
} 
decisions are publicly available on Légifrance. Therefore the decisions of the CNIL with respect to biometric systems constitute a very useful source of information to understand the criteria taken into account by a Data Protection Authority in the course of its authorizationmaking process. The goal of this research was to identify these criteria and to take them as a source of inspiration for building up the SALT framework.

A thorough selection and analysis of 458 deliberations (including authorizations and refusals) of the CNIL covering a 10 years period (2005-2014) has been carried out. All these deliberations have been classified according to context-related information and characteristics of the systems such as the activity of the requesting entity, the categories of people concerned, the type of system, the purpose, etc.

This systematic study has made it possible:

- to extract the essential criteria used in practice to assess the proportionality of a biometric system and

- to evaluate their respective weights in the decisions so as to use them in an automatic scoring process.

The main criteria identified by the study are the following:

- The categories of people to be enrolled in the system.

- The legitimacy of the system.

- The functionality of the system.

- The type of storage of the biometric characteristics.

Consistently with the new approach followed by the CNIL, the distinction between biometric leaving trace (such as fingerprints) or biometric leaving no trace (such as palm vein) has not been considered as critical because it is less and less technically relevant ${ }^{16}$.

\subsection{Mutual benefits}

The two results sketched in Section 5.1 and Section 5.2 are good illustrations of the benefits of the interdisciplinary approach because they could not have been obtained by separated teams of computer scientists, lawyers or social scientists. As anticipated in Section 3, one of the main sources of debate during the elaboration of the SALT framework was the level of automation and the types of interaction of the system with the different users. On one hand, the computer scientists designing the framework have been able to understand the needs expressed by the legal and social science partners and to integrate them in a way that is satisfactory for all members of the project. For example, the framework provides assistance rather than automated decision making, it includes links to legal contents, it allows for interactions with the users, etc. On the other hand, the legal and social science partners have been able to understand the possibilities offered by the techniques as well as their limitations and to adapt or rephrase their requirements so as to make them implementable.

The systematic study of the deliberations of the CNIL sketched in Section 5.2 illustrates another form of benefit from the interaction between disciplines. The result itself has a high value from the legal point of view and the systematic method applied to obtain it - in

\footnotetext{
16 Due to the development of the technologies, the frontier is blurring between these two types of biometrics.
} 
particular the extraction of the essential criteria and the evaluation of their respective weights in the decisions - can be seen as a form of reverse-engineering of a decision algorithm, which is familiar to computer scientists. In fact, the process itself, which relies on manual computations based on large decision tables, could give rise to a tool that could be useful in future studies.

To summarize:

- The SALT framework is a result in the field of computer science that is strongly influenced by lawyers and social scientists (and, thanks to this influence, is more interactive and less mechanical than it would have been otherwise)

- The study of the CNIL deliberations is a result in the field of law that is influenced by computer scientists (and, thanks to this influence, is more systematic than it would have been otherwise and could in the future lead to the design of a tool).

It should be clear from this summary that these results would have been obtained neither by a monodisciplinary approach nor by a simple multidisciplinary approach.

\section{Lessons to be drawn, recommendations}

As argued in a previous report on "Multidisciplinary research in FET", overcome the disparity in terms of scientific languages between people with different background is "time and intense communication to get rid of any major misunderstanding".

Based on the experience of the PARIS project, we would recommend:

- Conduct physical meetings with long sessions of open discussion, and no objective to converge or agree on a subject within the allocated timeslot: representatives of different disciplines do not have to align; they have to understand each other. It is often preferable that a result from an interdisciplinary group is accepted and stabilized (considered as agreed, at least temporarily) even if it is not perfectly defined or if slights disagreements or misunderstandings remain.

- Optimize the size of the group depending on the objective: social groups theory has shown that very small groups often disadvantage the expression of discrepancies, whereas too big groups have a natural tendency to split; the optimal size for collaborative working is often considered to be with 5 or 6 persons.

- Have a chairman for the meetings that stimulate convergence by proposing ideas, tools, examples, other directions. Concrete use cases are often useful to enhance mutual understanding and to find convergence points or new collaboration modes between the partners. It is important that he or she is not too directive, in order to really benefit from all the expertise of the group and to avoid the split of the group or the development of entrenched positions.

- When divergences are too strong, consider the introduction of a mediator (ideally within the project). The mediator could conduct point-to-point meetings and try to enhance mutual understanding and then present his conclusions to the whole project.

- Ensure that written reports are produced after each meeting. Intermediary drafts are key elements to make progress ("words vanish, writings remains") in a necessarily

17 Multidisciplinary research in FET, V. Gayraud, FET Trainee Report, 24/11/05. 
iterative process. Ideally, these reports should be written by experts of different disciplines to iron out the differences of style discussed in Section 3.

A sufficient amount of time should be devoted at the beginning of the project to the comparison of the terminologies and notions used in the different disciplines: often the same term is used in two disciplines with different meanings; sometimes the same notion can be named in different ways (e.g. "identifiability", "control", "security", "autonomy", "effectiveness", in the field of privacy). The comparison of these shifts is a pre-requisite for common understanding; in addition it can shed new light on each discipline and help refining the underlying concepts.

It is also important to understand the differences of procedures and operating modes in different disciplines: for example how are the instruments conceived, how are they accepted, monitored, revised ? This comparison, in addition to enhancing mutual understanding, can be a source of inspiration and improvement in each discipline. The two results presented in Section 5 are good illustrations of this benefit.

As far as the organization of the project is concerned, the four main recommendations are the following:

- First of all, a balance should be stroke between disciplines. The main pitfall to avoid is to reduce a discipline to the role of « service provider» to the other partners. Too many projects apparently suffer from this initial bias and do not give rise to truly interdisciplinary research because of this reason. This kind of project can be at best multidisciplinary but they are often frustrating for the partners that do not play a central role. Interdisciplinary can work only in "win-win" situations.

- The partners from different disciplines should all be involved within the main tasks and outcomes of an interdisciplinary project. A project with specific tasks per discipline is likely to be multidisciplinary rather than interdisciplinary. This choice is essential to foster collaboration between partners.

- The project should be iterative because the mutual understanding of the partners will increase over time and this should be taken into account in the overall structure of the project.

- Tasks should not be defined too precisely from the beginning because the interdisciplinary approach, if successful, will stimulate the creativity, it will bring new ideas and suggest new ways to tackle the problems. A too narrowly defined project would limit the benefits of the whole approach or lead the project to failure (if it turns out to be too difficult for a partner to fit within the pre-existing plan). Flexibility provides more freedom to shape the project in a collaborative way.

Last but not least, as pointed out in previous reports, the human component is crucial. Interdisciplinarity is time-consuming, the initial cost is high, and researchers following this path are often not fully rewarded for their efforts. Therefore it requires strong motivation, real curiosity for other disciplines, patience and, above all, an open mind. 